\title{
Narrative in psychiatry and psychotherapy: the evidence?
}

Jeremy Holmes North Devon District Hospital, Barnstaple, Devon

\begin{abstract}
Psychiatry is perhaps the most "narrative" of all medical specialties, but here as elsewhere clinical skills are in danger of being lost as evidence-based medicine becomes the dominant paradigm in medical culture. Psychotherapy is a quintessentially narrative discipline. Starting from an "attachment" perspective, the uses of narrative in psychotherapy are outlined. These include the importance of metaphor, story-telling, the search for event-scripts, and the role of "narrative competence" as a mark of psychological health. Life history research, the "adult attachment interview" and other research approaches to narrative in psychiatry and psychotherapy are described. The paper calls for an integration of narrative and evidence-based medicine.

(7 Med Ethics: Medical Humanities 2000;26:92-96)
\end{abstract}

Keywords: Narrative; psychotherapy; attachment

For every thesis there is an antithesis. The current ascendancy of evidence-based medicine, (EBM) for all its power and beauty, has created disquiet among some clinicians and researchers. Are randomised controlled trials, (RCTs) practice guidelines, national service frameworks and protocols really the end of the story, or is there still a place for clinical judgment, the importance of the doctor-patient relationship and the traditional emphasis on individual meanings and case histories? Evidence-based medicine has thrown up a counter-movement, less organised and influential perhaps, whose voice is still uncertain, but which nevertheless holds onto narrative and story as a bedrock of clinical practice. ${ }^{1}$ The purpose of this article is to review aspects of narrative based medicine (NBM) as they impact on psychiatry and psychotherapy, to consider briefly research aspects of narrative, and to look at ways in which EBM and NBM can complement each other both for the benefit of the individual patient and more widely.

Evidence-based medicine argues that medical practice should model itself on scientific method and that all interactions with patients should be guided by the falsifiability principle: only those interventions which have been shown by rigorous tests to be effective should be implemented; thus will the health of the population benefit, and the costs of health services to the public purse be justified. In itself this approach is unexeptionable - who could reasonably disagree - and yet many health workers feel that something vital to medical practice is left out in this uncompromising position. ${ }^{2}$

\section{The limitations of evidence-based medicine}

The narrative critique draws on a number of different empirical and philosophical strands. First, there is the uncomfortable gap between efficacy and effectiveness: many doctors prescribe treatments which do not meet the rigorous criteria of EBM, and conversely fail to deliver treatments which have been shown to work. In schizophrenia for example there is a continuing failure to deliver psychosocial interventions on a routine basis despite two decades of strong evidence for their efficacy. Doctors need more than published results of RCTs to change their practice: acquiring psychosocial skills for themselves, treating patients and their families, seeing the resulting changes that can come about (and those that cannot) are all necessary. Only when the evidence is backed by a story of a successful treatment which the doctor can see with his or her own eyes is it likely to be incorporated into day-to-day practice. We learn by doing, and doing involves a self-generated narrative in which we are active agents of change, shaping, and being influenced by the results of our activities. For MacIntyre ${ }^{3}$ we are our narrative: our sense of self is inextricably bound up with our life-stories and the meanings they have generated. Narrative is thus synonymous with training and learning.

Advocates of narrative argue that with all the huge advances of modern scientific medicine there has been a loss of "meaning" in our work and in what has been offered to the patient. But what do we mean by meaning? According to Quine ${ }^{4}$ meaning arises out of connectedness-we look up a word in the dictionary and are offered another set of words to which that word is connected, and they are in turn linked to another set of words and so on until we reach a web of meanings that comprise an entire language. Meaning in this sense can be linked to both metaphor and story. Metaphor is a fruitful collision or connection between apparently unrelated phenomena. Many of the metaphors that arise in psychiatry and psychotherapy connect bodily experience to the external world. For example, a middle-aged man whose business had failed and who had become depressed described himself as "gutted" as he spoke about looking round his empty warehouse. The clear link between the 
external event and his feelings, however painful, gave meaning to his pain and helped him cope with it. Similarly, stories are series of linked intentions and actions which create a sense of connection and agency: first this happened, then I did that, and the result was the other.

Evidence-based medicine is often described as "instrumentalist" in that it applies the scientific method within a given reality, but does not consider the historical and sociological forces which shape that reality. For example, resistance to the implementation of psychosocial interventions in schizophrenia arises in the context of a pharmaceutical industry which invests vast sums in order to influence doctors to prescribe its neuroleptic treatments, with psychosocial interventions coming in a very poor second in the list of priorities. The instrumentalist perspective of EBM posits the doctor as a scientific agent of change, offering his validated treatments to a passive patient. In NBM doctor and patient together are "co-constructors" of a shared reality in which the history and expectations of each play significant roles in determining outcome. For example, we need to know what our patients' attitude towards medication are-whether they are "anti-pills", have tried homoeopathy or have specific spiritual beliefs, tend to be forgetful, are obsessional etc-before gauging the likelihood of compliance with our prescriptions. These attitudes are all embedded in the patient's individual experience and encoded as a series of stories grandmother, say, who was given aspirin, developed a gastrointestinal bleed and died, or a religious teaching which fosters a passive acceptance of "fate"which comprise their personal narratives. Critics of EBM argue that modern medicine tends to downplay these vital aspects of medical practice, while the traditional skills of history-taking and listening may suffer in the face of the drive to examine, investigate and intervene. Good practice requires a blend of evidence-based and narrative approaches, in which scientific knowledge is adapted and tailored to fit with the particular circumstances, biography, and personality of each individual patient.

\section{Narrative and causality}

Supporters of EBM might be happy to concede the points made so far, but argue that what is being said is both obvious and trivial - of course we need to take histories carefully and respect patients and their beliefs, but the cutting edge of treatment remains based on evidence, not narrative. "What about Down's syndrome?" they might suggest. The narrative approach held sway until the discovery of trisomy in 1956: mothers of Down's syndrome babies were asked about physical and emotional trauma during pregnancy and were found to have a much greater incidence than mothers of nonaffected babies. ${ }^{5}$ Here a narrative explanation stood in the way of the "real" evidence: the overwhelming need to make sense of experience led these mothers to connect the trauma of their abnormal baby with previous trauma, whereas in reality there was no such link, and presumably mothers of normal babies had had equal amount amounts of unhappiness whose significance faded once their babies were born without blemish.

The argument here is essentially about causality. Bolton and $\mathrm{Hill}^{6}$ distinguish between "intentional" and "non-intentional" causality-the former implies agency of some kind, while the latter is outside the realm of human intention. Garland ${ }^{7}$ similarly distinguishes trauma that has a "man-made", intentional, aspect from traumas that are outside the realms of human agency. Down's syndrome turned out to be a "non-intentional" story about chromosomes, not human agency. But if we take Down's syndrome as a paradigm we will miss much that is central to medical and especially psychiatric practice-the interplay between intentional and non-intentional causality is where most problems lie. As a counterexample, if we simply collude with patients who attribute their depression to a "chemical imbalance in the brain" (which is not to deny that such "imbalance" exists), and fail to take account of the ways in which their illness arises out of their life-story, we will miss the role of loss and the associated grief which Brown and others have shown to be causative factors in the origins of depressive illness, ${ }^{8}$ and which, it seems likely, also plays a role in the expression of a genetic vulnerability to schizophrenia. Furthermore, even though Down's syndrome is a product of "nonintentional causality", there remains a story to be attended to about how a family copes with the advent of a handicapped child.

\section{The uses of narrative in clinical practice}

What is the role of narrative in clinical practice? Narrative methods are central to history-taking and formulation, to engagement, and to several aspects of psychotherapy.

HISTORY-TAKING AND FORMULATION

Psychiatric patients often present to services in bemused and de-contextualised states. A patient arrives in casualty after an overdose. A young person who has been behaving strangely is dragged along by anxious relatives. Someone known to be psychotic refuses to let his community worker into the house. The clinician's task is to find a story into which this fragment of inexplicable or worrying behaviour can be fitted. A story is always about the interplay of intention and context-or, to put it the other way round, when we have found a human agent acting within a context, then we have a story. In a clinical context, a story is a sequence of events centring on a suffering patient. It consists of alternating episodes of "what was done to me/what I felt", and "how I reacted/responded/felt about it in return”.

We often ask a patient on first meeting: "What's the story?", or "How did you land up here in this room talking to someone like me?", or "How did it all begin?". The aim is to reconstruct a narrative chain out of an apparently disconnected series of events, ending with the here-and-now, this patient talking to this doctor, in this room, on this day, 
about this subject-the story so far. By proceeding in this way we begin immediately to encourage the patient to become the author of her own story-to consider what has happened to her, how she has reacted, and what she was and is feeling about it We start from a feeling of puzzlement, akin to starting a novel or listening to a story: "Why did so-and-so meet so-and-so on such-and-such a day", "What is going to happen next", "How will it all end", and do not rest until we have some sort of picture of what the overall shape of the story might be. At first glance this will probably be fairly familiar: the overdose that followed a row with a boyfriend, the young man who has been smoking too much cannabis, the patient who has stopped taking his medication. But stories are infinite: each one leads onto another. Why did she react to a row in that way? Why did the row occur in the first place? Is this an example of separation anxiety in someone whose parents split up when she was a child? What led him to retreat into cannabis? Had he lost his job or his girlfriend? Did the cannabis make him depressed, or was it the other way round? Why did he stop his pills? Was it the side effects? Or did the hallucinations tell him to? Perhaps he only believes in homoeopathy? Is that a valid or a delusional belief? And so on. Thus a narrative approach to history-taking and formulation leads the listener deeper and deeper into the causal chain of eventsboth intentional and non-intentional - which underlie the presenting problem, and can be used to help patients put their illness in a context which helps give meaning to their apparently meaningless pain.

\section{ENGAGEMENT}

"Engagement", or therapeutic alliance, is the backdrop to the mutual commitment of doctor and patient to the understanding and tackling of the problem which the patient brings for amelioration. In psychotherapy the quality of the therapeutic alliance is the single best predictor of good outcome for treatment. ${ }^{9}$ A narrative approach can be a powerful method of engaging the patient, who encounters a clinician who genuinely wants to know how things look from his, the patient's, point of view, who wants to hear his, or a the very least his side of the story. Every good psychiatric clinician is driven by a fascination with the phenomenology of patients' experience, by the ramifications of their delusional system, the minutiae of their everyday lives-by a wish to understand this particular person's story, to get to the heart of her suffering - and accords this enterprise equal importance to the need to understand the mental or biochemical mechanisms involved. In working with psychotic patients, EBM will tell the clinician which antipsychotic drug is likely to be effective; but listening carefully to the details of the patient's psychotic experiences, and trying to draw out a narrative pattern in his story, is a precondition for successful prescription. Without the engagement which this implies the patient is far less likely to take his medication or to trust the clinician in other ways.

NARRATIVE AND SUPPORTIVE PSYCHOTHERAPY

A significant part of the effectiveness of psychotherapy is attributable to "common" or "nonspecific factors" which form part of the therapeutic process, whether psychoanalytic or cognitive. ${ }^{10}$ Supportive psychotherapy relies on this phenomenon for its usefulness. A basic technique in supportive psychotherapy consists simply of encouraging patients to tell their story, and to "ventilate" their feelings about the events of their life. Winnicott ${ }^{11}$ described psychoanalysis as an "extended form of history-taking"; offering patients a supportive relationship sustained over many years similarly comprises a significant part of the work of the consultant psychiatrist. The narrative stance requires a consistent curiosity on the part of the doctor, who always wants to hear more, and who learns to cultivate a creative puzzlement about aspects of the patient's story which do not seem to make sense.

The importance of this emergent "autobiographical competence" 12 has recently been supported by a study which showed that even physical illness can benefit from narrative activity. Patients with rheumatoid arthritis and asthma were asked to write about stressful experiences in their lives. Compared with controls who did not write, or simply wrote about neutral topics, their symptoms improved over a six-month period on a variety of objective measures. ${ }^{13}$ It is intriguing to speculate about the possible mechanisms which might explain this finding. One possibility is to think of writing or telling one's story as representing a form of surrogate relationship, albeit to oneself, with all the anxiety reduction and possibility for processing mental and physical pain that that entails. In other words, it can help create an internal sense of a "secure base". The objectification of feelings involved in the writing task helps the sufferer to distance herself from her pain. In addition, relationship implies connectedness, and an escape from the narcissism which chronic illness, whether physical or mental, can foster.

NARRATIVE AND ATTACHMENT

This links with the concept of "reflexive function" (RF) which has emerged from attachment research, ${ }^{14}$ suggesting that people who are able to "represent" and so reflect upon their experience in words, however problematic or painful, are more likely to be able to form secure attachments than those who lack such capacity. For the purposes of our discussion, this self-reflexive capacity is important in three ways.

First because it is exactly what psychotherapists aim to foster in their patients, whether they be cognitive therapists working with automatic thoughts and assumptions, or analytic therapists with their motto, "where id is there ego shall be". Ego is the psyche's story-teller. Second, in a general practice context, the 
very existence of a general practitioner (GP) and a surgery gives a message to the patient that his pain and difficulties can be "represented"-first talked about and then acted on so as to reduce suffering. Third, RF and narrative style generally have been shown to be linked with early relationship patterns. Longitudinal studies have shown that children who are securely attached in infancy are more likely to have a coherent and "free-autonomous" narrative style when talking about themselves in early adulthood, as compared with insecurely attached children whose narratives tend either to be overelaborated and confused (linked with ambivalent attachments) or sparsely dismissive (linked with the avoidant attachment pattern). Thus the ways in which people talk about themselves reveal fundamental relationship patterns.

This insight into narrative styles can enhance the quality of listening with which we approach patients, and guide our interventions in "storymaking" or "story-breaking" directions. ${ }^{1}$ Patients with self-sufficient, unelaborated, dismissive narratives need to be encouraged to break open their defensive stories and consider other possibilities. Those who seem unable to find a narrative thread and to be drowning in the chaos of their experience need to be helped to find a shape and a pattern which helps fit things into place. Narrative style, in other words, can be a guide to diagnosis, pointing to particular relationship patterns in childhood, and propensities in adult life.

\section{Narrative and metaphor in dynamic psychotherapy}

Dynamic therapy is redolent with metaphor. ${ }^{12}$ The word metaphor and transference, although from Greek and Latin roots respectively, are etymologically the same, referring to carrying something across, and therefore, by extension, to the making of connections. Hobson's ${ }^{15}$ Conversational Model or Psychodynamic-Interpersonal therapy explicitly encourages patient and therapist to explore feelings using metaphor. A metaphor is a fundamental narrative device: a memorable image that gives meaning to the patient's difficulty, a "third term" which helps a patient to begin to objectify her problems. It links together different aspects of the patient's life, and is open to discussion, modification, or elaboration by both patient and therapist. In a Winnicottian sense ${ }^{11}$ it lies "transitionally" between patient and therapist, with a life of its own, and is not wholly the property of either. As a "third term" a metaphor belongs to the "oedipal" stage of development, where the child has both to cope with the triangle of mother, father, and herself, but also gains the objectivity which being able to observe the parental couple from the outside brings. Metaphor thus simultaneously pulls patient and therapist together and separates them from the lure of narcissistic fusion or collusion.

As a fundamental narrative device metaphor and simile can be compared with the scientific hypotheses of EBM. Both represent speculations which need to be tested against reality. For EBM the cri- teria are objective; for therapists the main test of their metaphors is whether they "feel" right to themselves and their patients. Unlike a scientific hypothesis metaphor is always specific to a given situation-it upholds the uniqueness of the individual life-history. Its terms are not interchangeable in the way that in an equation, for example, the number 3 can always be substituted by the square root of 9 to give the same result. People are troubled by a fairly limited range of problems, centring on the biological fundamentals of relatedness- "birth, copulation and death" " - but one life-history is never exchangeable with another. We cannot create a composite Dickens novel by replacing Pip by Martin Chuzzlewit in Great Expectations. This is a basic difference between EBM, which is based on the behaviour of populations whose elements are equivalent, and NBM, which is always about particular cases. This is not to say, however, that therapeutic metaphors are entirely subjective. Psychotherapy research methods can measure the impact of therapist utterances in terms of patient speech patterns or emotional responses, and thus get some indication of the therapeutic impact of a metaphor. ${ }^{10}$

The narrative thrust in dynamic psychotherapy is always patient-centred. The role of the therapist is as "auxiliary autobiographer", helping the patient to clarify the contours of his own story and to make sense of it. A similar practice at the level of the family is to be found in systemic therapy. ${ }^{17}$ Here the effort is to track "family scripts", the unconscious rules and expectations which shape family life across generations and which trap individual members into painful or self-defeating roles and behaviours.

Some family therapists go further than this and use story-telling and jokes as a therapeutic device in its own right. For example if a couple are rowing in a session, the therapist might intervene with the well-known joke "is this a private fight, or can anyone join in?". Stories here are equivalent to the generalisations and laws of EBM-universal psychological truths which can help people cope with mental pain. The informal subculture of medicine abounds with such stories. ${ }^{18}$ "Balint groups" for trainee psychiatrists are one way to legitimise doctors' need to look at their own stories when faced with the emotional impact of their work. In one such group it emerged that as the junior doctors were lunching together someone had proposed a competition for the most absurd form of deliberate self harm encountered during the past six months; these ranged from attempting to gas oneself with carbon monoxide in an open cabriolet, to hanging oneself using the emergency bell pull. While this was undoubtedly disrespectful to the patients concerned, it also expressed the tremendous strain which young doctors experience in the face of their patients' death wishes-a story in its own right, which also needs to be told. Here too NBM recognises and celebrates this aspect of practice, complementing, enhancing and helping to deliver effectively the many contributions of contemporary scientific medicine. 
Stories we remember-true or false?

The recovered memory debate has raged furiously in psychotherapy recently and cannot be ignored in any discussion of NBM. There are those who claim that false memories can be implanted in suggestible subjects by unscrupulous psychotherapists, while on the other side there is much evidence to suggest that painful memories can be obliterated from memory, only to surface at times of stress or further trauma. The key issue for the discussion of narrative is that stories should not necessarily be taken at face value. Indeed it is often a mark of an inexperienced therapist to attend too closely to "content" and to miss the tone, context, and interactive dimension of what is being said. Stories always exist in an interpersonal setting: there is a story-teller and a listener, and the story needs to be understood in terms of their relationship. Therapists should ask themselves why is this person telling me this story, in this way, on this occasion? They should also be able to tolerate the uncertainty of not knowing how true any particular story happens to be. While some issues are indeed matters of simple fact - was this person sexually abused or not-more often memories are questions of tone and perspective. As already mentioned, some patients need help to create a coherent account of what has happened to them (story-making), others to consider other possibilities than the simplistic account to which they cling (story-breaking). In the course of treatment peoples' view of themselves and their history will change. The past is reconsidered in the light of the present. The "true story", to recapitulate, is a pattern of agency and context. The therapist's task is to help patients find a story that pays due attention to both - to what they have made of what they are made of.

\section{Research in NBM}

Although narrative-based medicine may not sit comfortably with conventional EBM methodologies, especially the RCT, narrative is far from unresearchable. In the course of this article three approaches to systematic study of narrative have already been mentioned. Brown's ${ }^{7}$ studies of depression over three decades in women rely on narrative methods in which verifiable events are evaluated by researchers who then look at the interpersonal context of the sufferer and make judgments about how loss and difficulty has impacted on individual lives. The Adult Attachment Interview (AAI) ${ }^{19}$ is a quintessentially narrative instrument which takes transcripts of interviews and subjects them to structural and linguistic analysis, resulting in reliable codings of narrative style, which in turn link with attachment patterns. Luborsky's Core Conflictual Relationship Theme approach ${ }^{20}$ similarly relies on interview transcripts to extract narrative episodes which are then classified according to predetermined interpersonal features. This measure of psychotherapy process can then be used in a variety of ways, for example to look at correlations between outcomes and, say, to focus on transference in the content of the sessions.

\section{Conclusions: towards integration}

I have tried in this article to argue the case for NBM, both theoretically and as central to the everyday practice of psychotherapeutic psychiatry. Conventional medicine has a tendency to be conservative, and to be at times self-serving. Evidence-based medicine is a necessary spur which questions traditional assumptions and values. There is no inherent conflict between narrative and evidence-based approaches: understanding the patient's story helps align scientific knowledge with the specific needs and predilections of individual patients. But in an ever-accelerating culture we run the danger of losing touch with a historical and developmental perspective which reminds us that the present has evolved out of the past. As psychiatrists it is simply not good enough to seek for the symptoms of schizophrenia, prescribe the latest antipsychotic, and feel that the job is done. We need to attend as closely to the patient's story and context as we do to the minutiae of the mental state examination. Evidence-based medicine and NBM are complementary facets of a wider whole. Finding the ways in which they intersect is an urgent task for the next generation of psychiatrists.

feremy Holmes is a Consultant Psychiatristl psychotherapist at the North Devon District Hospital, Barnstaple,Devon.j.a.holmes@btinternet.com

\section{References}

1 Roberts G, Holmes J. Healing stories: narrative in psychiatry and psychotherapy. Oxford: Oxford University Press, 1998.

2 Greenhalgh T, Hurwitz B. Narrative based medicine. London: BMJ Publications, 1999.

3 Macintyre A. After virtue. Notre Dame, USA: University of Notre Dame Press, 1981.

4 Caper R. A mind of one's own. London: Routledge, 1999: 129. 5 Bouras N. Mental health in mental retardation. Cambridge: Cambridge University Press, 1994.

6 Bolton D, Hill J. Mind, meaning and mental disorder. Oxford:

7 Gxford University Press, 1996. London: Duckworth, 1998.

8 Brown G, Harris T. The social origins of depression. London: Brown G, Harris
Tavistock, 1979.

Tavistock, 1979.
Luborsky L. A pattern-setting therapeutic alliance study revisited. Psychotherapy Research 2000;10:17-29

10 Frank J. Persuasion and healing. Baltimore: Johns Hopkins University Press, 1961

11 Winnicott $\mathrm{D}$. The maturational processes and the facilitating environment. London: Hogarth, 1968.

12 Holmes J. Between art and science. London: Routledge, 1992.

13 Greenhalgh T. Writing as therapy. BMF 1999;319:270-1.

14 Fonagy P. Psychoanalytic theory from the viewpoint of attachment theory and research. In: Cassidy J, Shaver P, eds. Handbook of attachment. New York: Guilford Press, 1999.

15 Hobson R. Forms of feeling: the heart of psychotherapy. London: Routledge, 1985

16 Eliot TS. A family reunion. London: Faber, 1937.

17 Byng-Hall J. Creating a coherent story in family therapy. In: Roberts G, Holmes J, eds. Healing stories. Oxford: Oxford University Press, 1998.

18 Hunter K. Doctors' stories: the narrative structure of medical knowledge. Princeton: University of Princeton Press, 1991.

19 See reference 14: Hesse N. The adult attachment interview.

20 Luborsky LK. Principles of psychoanalytic psychotherapy. New Luborsky LK. Principles
York: Basic Books, 1984. 